\title{
Near-Simultaneous Core- and Low-Loss EELS Spectrum-Imaging in the STEM using a Fast Beam Switch.
}

\author{
P. J. Thomas*, J. Scott**, M. MacKenzie**, S. McFadzean**, J. Wilbrink* and A. J. Craven** \\ * Gatan Research and Development, 5933 Coronado Lane, Pleasanton, CA 94588 USA \\ ** Department of Physics and Astronomy, University of Glasgow, Glasgow G12 8QQ, UK
}

The ability to simultaneously capture multiple signals with high spatial resolution makes the STEM spectrum-imaging (STEM-SI) technique a powerful tool for materials characterisation. In particular, the EELS signal contains a wealth of information regarding the compositional, chemical, physical and electronic properties of the material under analysis [1]. This information spans both the low and core-loss regions of the EELS spectrum. However, despite the relatively large dynamic range of modern electron detectors, it is still difficult to acquire both of these components under identical electron-optical conditions. This difficulty arises from the high intensity of the zero-loss, which is typically many orders of magnitude higher than that of the weak ionisation edges of interest.

A solution to this problem is to use a fast beam switch, allowing the low- and core-loss spectra to be acquired sequentially under identical conditions with the low-loss intensity attenuated to an appropriate level using a short exposure time [2]. Here, we report an extension to this approach to allow both core- and low-loss EELS spectra to be acquired as a STEM spectrum-image. The hardware configuration used is shown schematically in Fig.1a. Briefly, it comprises of a STEM equipped with a Gatan Enfina post-column EELS spectrometer, with additional signals provided by an ADF and an EDS detector. A custom-built electrostatic shutter provides fast beam blanking, which is capable of exposure times as short as $1 \mathrm{~s}$. The spectrum-image acquisition itself is controlled using Gatan's DigitalMicrograph software, and proceeds by acquiring a core and low-loss spectrum at each pixel in the spectrum-image. Core-loss spectra are acquired with beam shuttering close to the frame time of the camera (e.g. 100ms), whilst the low-loss is captured using a fast beam exposure (e.g. 1ms). Between spectra, energy-shifting of the EELS spectrum is performed at highspeed using the spectrometer's electrostatic drift-tube and, throughout acquisition, precautions are taken to maintain synchronisation between the spectrometer detector, fast shutter, beam position and spectrometer energy-loss state to avoid any signal mixing or detector over-exposure (Fig. 1b).

A preliminary data set acquired using the system is shown in Figs.1c-e, taken from a high-k dielectric gate stack. The acquired core- and low-loss EELS spectrum-images, along with the ADF signal captured simultaneously, are shown in Fig.1d. Extracted spectra are shown overlaid in Fig.1e and, as can be seen, the two complementary signals provide signal levels suitable for quantification from $0-700 \mathrm{eV}$ loss and beyond. Post acquisition, the data can easily be spliced together suitable for absolute quantitative analysis, as illustrated in Fig. 1f. These and further results will be presented to illustrate the advantages and future potential of this approach to STEM-SI acquisition [3].

\section{References}

[1] R.F. Egerton, Electron Energy Loss Spectroscopy in the Electron Microscope, $2^{\text {nd }}$ Ed, Plenum, New York, 1996

[2] A.J. Craven, J.A. Wilson, and W.A.P. Nicholson, Ultramicroscopy 92 (2002) 165

[3] Ray Pallister and Bernd Kraus are gratefully acknowledged for their contribution to this work. 
(a)

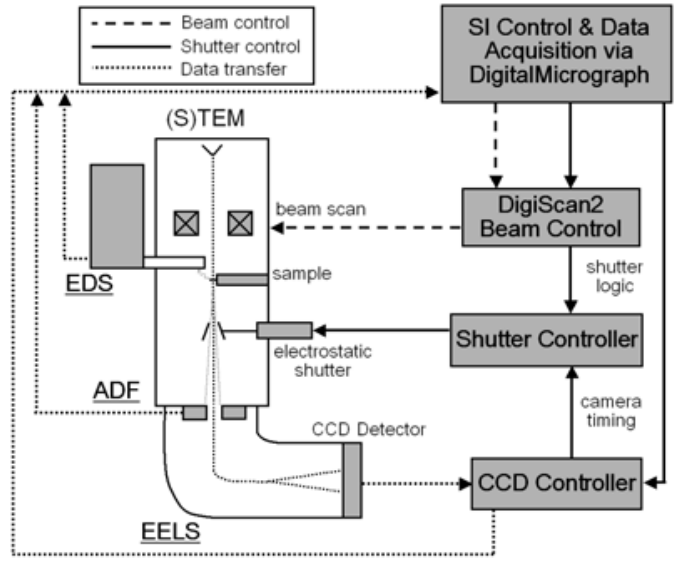

(c)

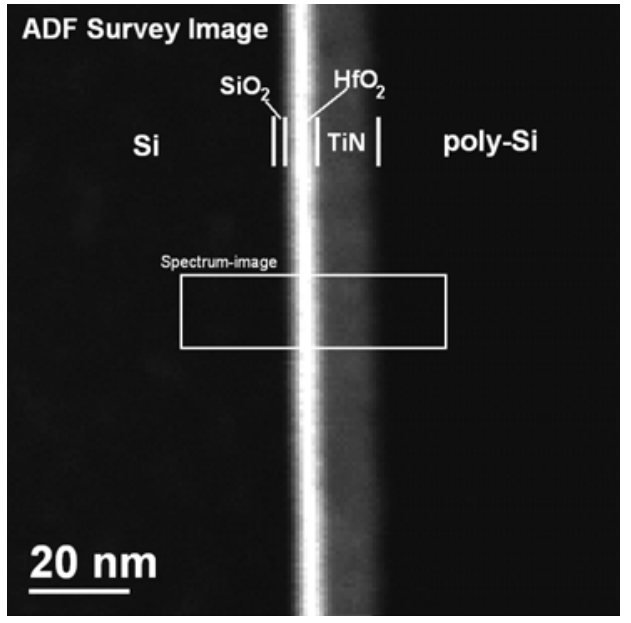

(e)

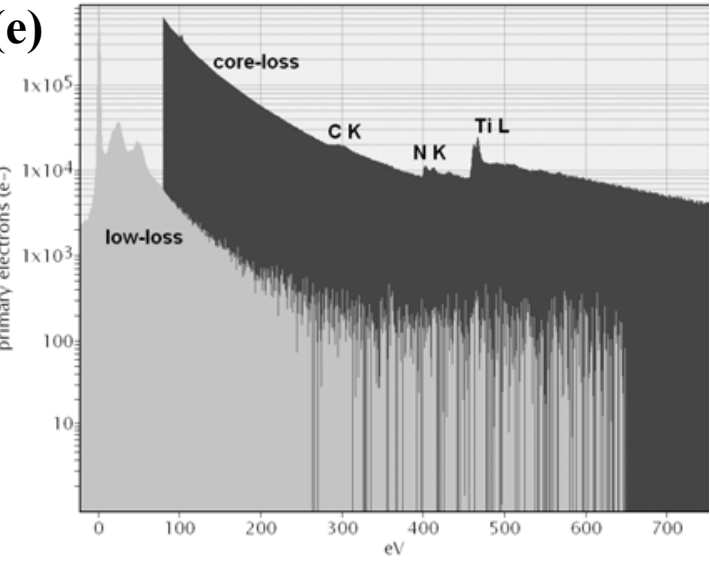



(d)
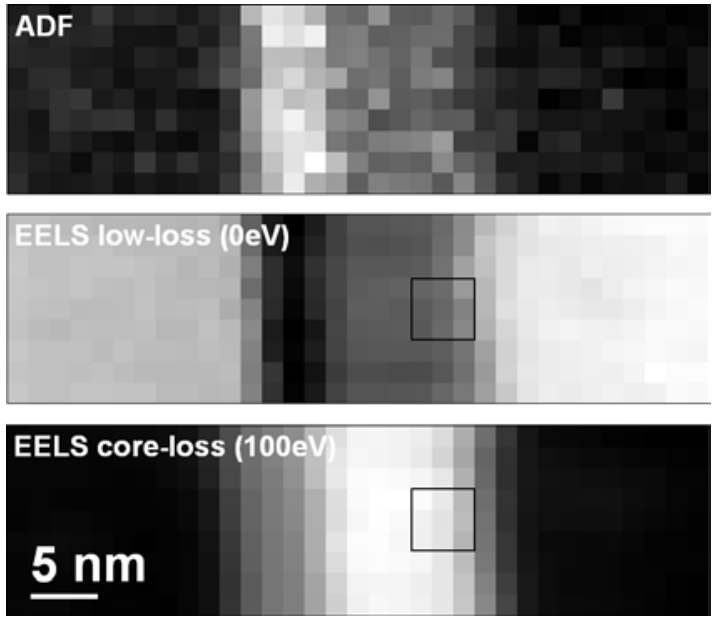

(f)

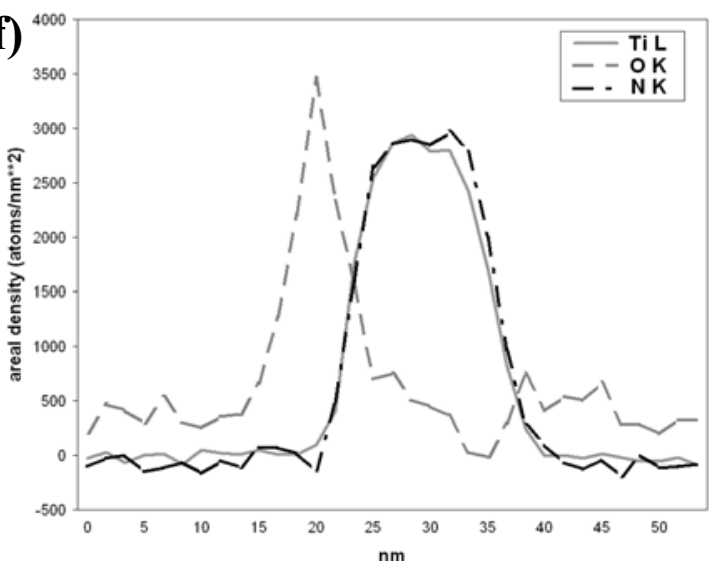

Fig.1. (a) Schematic diagram showing the hardware configuration used. (b) Timing diagram showing the synchronization of the beam scan, spectrometer energy-shift and electrostatic shutter signals for typical acquisition conditions. (c) ADF survey image of the high-k dielectric gate stack. (d) Signals acquired from the rectangle marked on c. The core-loss spectrum image was captured using an effective integration time of $70 \mathrm{~ms}$ per spectrum, and the low-loss with $0.77 \mathrm{~ms}$. (e) Lowand core-loss EELS spectra extracted from the regions indicated in d, displayed overlaid with logarithmic scale for comparison. (f) Atomic areal density profiles computed from the data for Ti, N and $\mathrm{O}$ across the interface. The spectrum-images were spliced and plural scattering removed by Fourier-log deconvolution prior to performing absolute quantification. 